\title{
Koebner Phenomenon Delays Therapeutic Effect of Pulsed Dye Laser on Refractory Psoriasis
}

Jae Wan Park

Sun Hye Shin

Hye Sung Han

Kwang Ho Yoo

Department of Dermatology, Chung-Ang University College of Medicine, Seoul, Korea
Psoriasis is a common chronic inflammatory skin disease that is histopathologically characterized by abnormal differentiation and hyperproliferation of keratinocytes, infiltration of $T$ lymphocytes, and alternations of the dermal vasculature. Pulsed dye laser (PDL) is commonly used to treat cutaneous vascular lesions, and it has been studied for the treatment of localized psoriatic lesions. A 30-year-old female patient with refractory psoriasis on her forehead was treated using PDL. After two sessions, the size of the psoriatic lesion increased, which might have occurred due to koebnerization. As the patient continued to receive PDL treatment, the lesion showed significant improvement with no recurrence on the one-year follow-up. We present here a case of refractory psoriasis treated with PDL and demonstrates an association between a delayed therapeutic effect and the Koebner phenomenon.

\section{Key words}

Koebner phenomenon; Psoriasis; Pulsed dye laser 


\section{INTRODUCTION}

Pulsed dye laser (PDL) has a wavelength of 585-595 $\mathrm{nm}$, which targets the chromophore hemoglobin; it can selectively damage blood vessels and was first used to treat psoriasis in 1992 by Hacker and Rasmussen. ${ }^{\text {P PDL }}$ provides optimal outcomes for nail psoriasis when used as the second-line therapy. Furthermore, in recent years, it has been used to treat non-vascular indications, such as psoriasis. Erceg et al. reviewed the efficacy of PDL for inflammatory skin disease and concluded that it is an effective and safe method for treating localized plaque psoriasis. ${ }^{2}$ Although light-based modalities (including PDL) are convenient for use in small areas, their therapeutic effects were not confirmed until 13 weeks. ${ }^{3}$ This may suggest that skin lesions may be aggravated during the early treatment phase due to the Koebner phenomenon, with treatment effects emerging later, contrary to expectations. We present our experience with this phenomenon in one of our patients.

\section{CASE REPORT}

Our patient was a 30-year old pregnant female with a multi-year history of plaque psoriasis. She had been treated with both topical betamethasone dipropionate with calciportriol (Enstilum Foam ${ }^{\circledR}$; LEO Pharma; Seoul, Korea) and systemic agents (cyclosporine, $150 \mathrm{mg} /$ day) and narrow-band ultraviolet B phototherapy. She sought treatment for her forehead lesion, which seemed refractory to previous treatment, because of her impending wedding ceremony and photography. We decided to treat the lesion with PDL $(595 \mathrm{~nm}$; spot size, $7 \mathrm{~mm}$; pulse duration, $0.45 \mathrm{~mm}$; fluence, $4.0 \mathrm{~J} / \mathrm{cm}^{2}$ ). Each session was separated by a three-week interval. Initially, the symptoms worsened, and the lesion increased slightly in size throughout the first two treatment sessions (Fig. 1A). However, starting with the third treatment session, her lesion size decreased, and the scaly patches continued to improve over six sessions. Ultimately, the lesion showed significant improvement (Fig. 1B) and had not recurred by her one-year follow-up appointment (Fig. 1C).

\section{DISCUSSION}

Laser therapy can be used, alone or in combination, to treat refractory psoriatic lesions. PDL has been used to treat psoriasis because of the highly vascular nature of psoriatic lesions. Previous studies suggested that the therapeutic effects of PDL arise from both the selective photo-thermolysis effect of hemoglobin in the vessels and immune-related mechanisms. Mechanistically, the levels of vascular endothelial growth factor receptor 2, 3, and E-selectin protein were reduced in lesions after PDL treatment, followed by down-regulation of tumor necrosis factor- $\alpha$ and interleukin-23p19. The authors speculated that PDL mainly acted on the capillaries within the upper dermis. ${ }^{4}$ PDL also reduced the number of activated and memory effector T-cells in the dermis and cytotoxic $T$ cells in the epidermis in patients with plaque psoriasis.

Our patient's scaly plaques started spreading after the first two treatment sessions. The new lesion disappeared once PDL treatment continued for three treatment sessions. Even though the cause was not clear, a possible mechanism of new lesion development might have been koebnerization. Though the pathogenesis of the Koebner phenomenon is still obscure, two steps are thought to be essential: a nonspecific inflammatory step and diseasespecific reactions. ${ }^{5}$ It is considered that PDL might be trauma, inducing inflammatory and further immune reac-
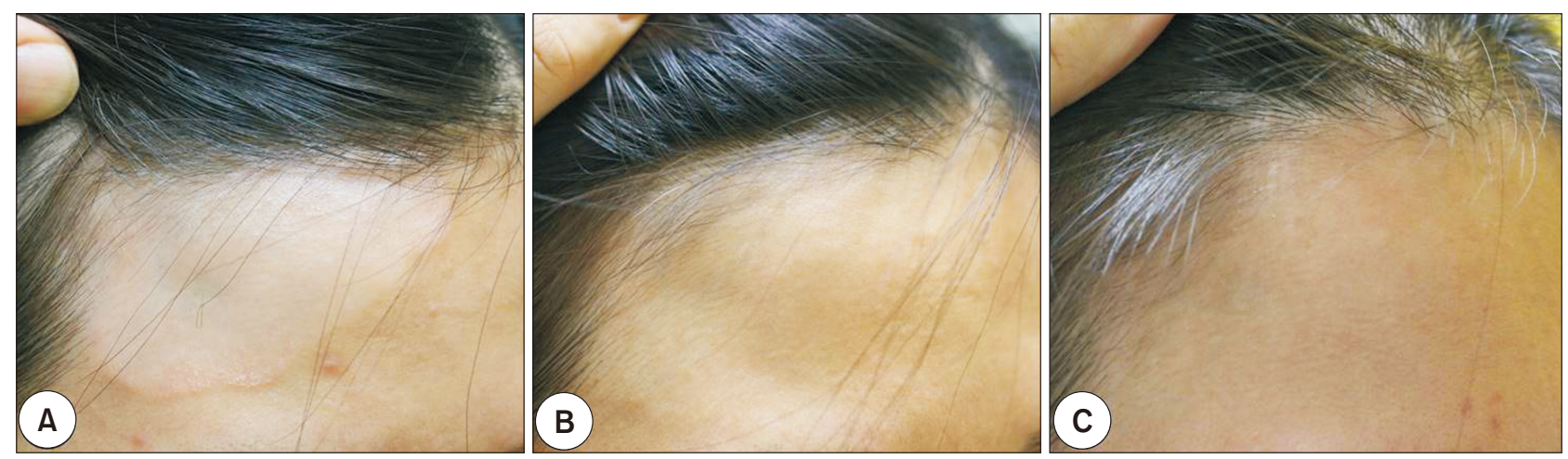

Fig. 1. (A) After 2 sessions of pulsed dye laser (PDL) treatment. The size of the lesion increased. (B) After 6 sessions of PDL treatment. The lesion showed significant improvement. (C) The lesion did not recur 1 year after PDL treatment. 
tions, and concurrently therapeutic effect, as mentioned above. So far, there are no reports of lesion spreading or signs of koebnerization after PDL treatment.

Pulsed dye laser should be considered as a potential therapy for psoriasis in patients with localized, refractory lesions, where it was not previously used as the primary treatment method because of pregnancy or other conditions. Furthermore, clinicians should recognize the possibility of koebnerization, which could explain delays in the therapeutic effects of PDL.

\section{CONFLICT OF INTEREST}

The authors have no conflicts of interest to declare.

\section{REFERENCES}

1. Hacker SM, Rasmussen JE. The effect of flash lamp-pulsed dye laser on psoriasis. Arch Dermatol 1992;128:853-5.
2. Erceg A, de Jong EM, van de Kerkhof PC, Seyger MM. The efficacy of pulsed dye laser treatment for inflammatory skin diseases: a systematic review. J Am Acad Dermatol 2013;69:60915.e8.

3. De Leeuw J, Van Lingen RG, Both H, Tank B, Nijsten T, Martino Neumann HA. A comparative study on the efficacy of treatment with $585 \mathrm{~nm}$ pulsed dye laser and ultraviolet B-TL01 in plaque type psoriasis. Dermatol Surg 2009;35:80-91.

4. Zhang P, Wu MX. A clinical review of phototherapy for psoriasis. Lasers Med Sci 2018;33:173-80.

5. Sagi L, Trau H. The Koebner phenomenon. Clin Dermatol 2011;29:231-6.

How to cite this article: Park JW, Shin SH, Han HS, Yoo $\mathrm{KH}$. Koebner phenomenon delays therapeutic effect of pulsed dye laser on refractory psoriasis. Med Lasers 2020; 9:62-64. https://doi.org/10.25289/ML.2020.9.1.62 